\title{
"VOTRE ENFANT DANS MA CLASSE". QUEL PARTENARIAT PARENTS-ENSEIGNANTE À L'ISSUE DU PREMIER ENTRETIEN ?
}

\author{
Laure Scalambrin et Tania Ogay
}

De Boeck Supérieur | Education et sociétés

$2014 / 2-n^{\circ} 34$
pages 23 à 38

ISSN 1373-847X

Article disponible en ligne à l'adresse:

http://www.cairn.info/revue-education-et-societes-2014-2-page-23.htm

Pour citer cet article :

Scalambrin Laure et Ogay Tania, « "Votre enfant dans ma classe". Quel partenariat parents-enseignante à l'issue du premier entretien ? »,

Education et sociétés, 2014/2 n³4, p. 23-38. DOI : 10.3917/es.034.0023

Distribution électronique Cairn.info pour De Boeck Supérieur.

(c) De Boeck Supérieur. Tous droits réservés pour tous pays.

La reproduction ou représentation de cet article, notamment par photocopie, n'est autorisée que dans les limites des conditions générales d'utilisation du site ou, le cas échéant, des conditions générales de la licence souscrite par votre établissement. Toute autre reproduction ou représentation, en tout ou partie, sous quelque forme et de quelque manière que ce soit, est interdite sauf accord préalable et écrit de l'éditeur, en dehors des cas prévus par la législation en vigueur en France. II est précisé que son stockage dans une base de données est également interdit. 


\title{
"Votre enfant dans ma classe". Quel partenariat parents-enseignante à l'issue du premier entretien?
}

\author{
Laure SCALAMBRIN \\ Département des Sciences de l'éducation, Université de Fribourg \\ Rue de Faucigny 2, 1700 Fribourg, Suisse \\ <laure.scalambrin@unifr.ch> \\ Tania Ogay \\ Département des Sciences de l'éducation, Université de Fribourg \\ Rue de Faucigny 2, 1700 Fribourg, Suisse \\ <tania.ogay@unifr.ch>
}

endant longtemps a dominé l'idée -notamment dans l'école républicaine
française- d'une coupure nécessaire entre l'institution scolaire et les familles, particulièrement les familles ouvrières ou populaires, afin d'extraire l'enfant de l'influence morale et culturelle de l'Église d'une part, de son milieu communautaire et familial d'autre part (Perroton 2000, Périer 2005). Depuis sa création et jusque dans les années 1950, les parents étaient tenus à l'écart de l'école publique obligatoire qui fonctionnait alors comme un monde clos et hostile pour ces familles, cloisonnées dans leurs quartiers ouvriers (Delay 2013). Les occasions de rencontre entre monde scolaire et univers domestique sont ponctuelles, se déroulant "dans un cadre social défini, voire ritualisé, et rarement "pédagogique" (de Queiroz 2000, 41). Les trois décennies qui suivent marquent progressivement le renforcement des liens entre ces deux instances de socialisation (Périer 2005). Un rapprochement devenu nécessaire pour lutter contre l'échec scolaire. Un changement de paradigme s'opère alors dans les discours officiels qui invitent les professionnels de l'éducation à collaborer avec les familles. Quant aux parents, constitués en partenaires de l'école, ils sont encouragés à rencontrer les enseignants de leur propre initiative et non uniquement sur convocation (Delay 2011). En Suisse, cette nouvelle politique de proximité s'affirme seulement depuis les années 2000. Constituée en norme institutionnelle, la relation entre l'école et les familles se construit dorénavant autour de ce principe qui, malgré les intentions généreuses et les significations positives et égalitaires, annonce un modèle de relations exigeant, complexe, voire hautement contradictoire (Périer 2005, Delay 
2011, 2013). Comment construire aujourd'hui un partenariat lorsque pendant longtemps a prévalu une relation entre les familles populaires et l'école soumise à un habitus de séparation?

\section{Revue de la littérature et questions de recherche}

D es recherches ont mis en évidence l'importance de la qualité des relations entre les familles et l'école dans la constitution de la réussite scolaire, en particulier pour les élèves issus de familles éloignées de la culture légitime (Bloom 1981, Léger \& Tripier 1986, Payet 1995, Dubet 1997, Terrail 1997, Thin 1998, Jaeggi et al. 2003, Henderson \& Mapp 2002, Périer 2007, Vatz-Laaroussi et al. 2008, Powell et al. 2010). Un projet de l'OCDE l'illustre où l'amélioration des relations entre l'école et les familles migrantes est une des dix mesures à mettre en œuvre pour résoudre le lancinant problème de la moindre réussite scolaire de ces élèves (Field et al. 2007). Familles et acteurs de l'école sont appelés à collaborer et à établir un partenariat (Pithon et al. 2008, Glasman 1992), voire une coéducation (Humbeeck et al. 2006, Rayna 2010), notion souvent employée dans le champ de la petite enfance.

L'éducation du jeune enfant bénéficie aussi d'un intérêt nouveau autant de la part des chercheurs que des décideurs de l'éducation, même s'il s'est fait attendre en Suisse (Stamm 2009, Pagnossin 2010). Un investissement est maintenant reconnu comme bénéfique aux enfants de familles dites minoritaires, notamment migrantes et socio-économiquement défavorisées. Cette reconnaissance a suscité des initiatives tel le programme "Encouragement de l'intégration dans le domaine préscolaire" financé par l'Office fédéral des migrations et la Commission fédérale pour les questions de migration (ODM/CFM 2012).

La réalisation d'une école fondée sur les principes d'égalité et d'équité étant souhaitée par les cantons suisses francophones en 2003 (CIIP 2003), il devient opportun de s'intéresser à la relation entre les familles et l'école dès les toutes premières années de scolarité. Si la production scientifique dans le domaine des relations familles-école est vaste, peu d'écrits renseignent sur les manières dont se construisent, s'articulent et s'ajustent les logiques et pratiques socialisatrices et éducatives entre parents et enseignants dès les premières années d'école. Fautil attendre qu'arrivent les devoirs à domicile pour s'intéresser à la collaboration familles-école ? Une part importante des recherches sur leurs relations traite de l'implication des parents dans la scolarité de leurs enfants et du soutien qu'ils apportent à ceux-ci pour les devoirs à domicile (Hoover-Dempsey et al. 2001). Ce qui se construit en amont, dès l'entrée à l'école lors des rencontres entre les familles et les enseignants dans cette configuration ponctuelle et institutionnalisée 
que sont les entretiens parents-enseignants (MacLure \& Walker 2000, Howard \& Liponoga 2010, Elbers \& de Hann 2014) reste encore largement inexploré.

L'entrée dans le cursus scolaire est un moment clé de transition (RimmKaufman \& Pianta 2000) entre le monde de la famille et celui de l'école où sont posées les bases d'une relation appelée à durer (Kherroubi 2008). Parents et acteurs de l'école doivent se côtoyer et travailler ensemble sur un objet commun, l'éducation de l'enfant (Mangez et al. 2002). L'entrée à l'école est aussi un moment clé pour les chercheurs, car, comme le relève Darmon (2001, 517), il s'agit d'une "entrée privilégiée" pour saisir les socialisations familiale et scolaire en jeu. Ces premiers échanges entre la famille et l'institution scolaire sont intéressants, car "les résultats de l'inculcation familiale sont appréciés de la manière la plus englobante" (Chamboredon \& Prévot 1973, 324), allant de la sociabilité de l'enfant, son développement cognitif, sa sensibilité à son épanouissement personnel et son état de santé. L'entrée dans le cursus scolaire coïncide pour les parents avec celle d'un nouvel acteur potentiellement capable d'émettre des critiques sur leurs pratiques éducatives et d'imposer des conceptions d'éducation à leur enfant (Mangez et al. 2002). Pour toutes les familles, mais surtout pour les familles de groupes minoritaires, l'entrée de leur enfant à l'école est une confrontation avec une nouvelle culture, impliquant une dynamique acculturative (Perregaux et al. 2008). La signification du partenariat à construire peut ainsi considérablement varier entre les acteurs, parents et enseignants.

Comment ces familles entament-elles ce processus de collaboration ? Comment construisent-elles, dans leurs interactions avec les enseignants, une relation où chacun apporte sa contribution à l'éducation de l'enfant ? Comment cette relation de proximité prend-elle forme, se noue-t-elle, se négocie-t-elle? Avec quelles ressources, quels ajustements et quelles attentes de part et d'autre? Pour tenter de répondre, cet article se réfère aux premiers résultats de deux recherches ethnographiques portant sur la construction de la relation de partenariat entre les familles minoritaires, en particulier migrantes et de conditions modestes, et les enseignantes durant la première année de scolarité. L'observation des premiers entretiens entre les enseignantes et les parents montre qu'ils y découvrent le regard porté par l'enseignante sur leur enfant et, au-delà, celui de l'institution scolaire.

Ces observations mettent au jour deux logiques intimement liées : la délivrance aux familles par les acteurs de l'école de ce qui s'apparente à des devoirs scolaires et à des conseils d'éducation de l'enfant, l'hypothèse étant que ces recommandations enseignantes sont liées à leur perception déficitaire des familles minoritaires (Boulanger et al. 2010), surtout si les conceptions éducatives familiales entrent en contradiction avec les référentiels éducatifs dominants. Le premier entretien parents-enseignante est ici appréhendé dans une multidimensionnalité : espace dialogique ambivalent, de prescriptions institutionnelles et de tensions normatives. 
En cherchant à mettre en évidence la triple dimension de la rencontre formelle où s'ancrent des échanges ambigus, la relation est traitée avant tout du point de vue des enseignants et de leurs pratiques professionnelles.

\section{Deux situations et deux recherches sur la construction de la relation familles-école}

L 'ethnographie par l'observation des premiers entretiens parents-enseignants constitue le cadre méthodologique des deux recherches mises en perspective ici. Elles visent à mieux comprendre la rencontre entre les familles de groupes minoritaires et les acteurs de l'école, saisie dès l'entrée à l'école. Ces deux enquêtes sont menées dans un établissement scolaire du canton de Genève et dans un établissement du canton de Fribourg.

La mise en place d'un principe de partenariat dans le canton de Genève est récente : il prend forme en 2005. La direction du Département de l'Instruction publique (DIP) inscrit treize priorités à son programme, dont "l'autonomie de l'établissement et partenariat avec les familles" (DIP 2005). À la rentrée scolaire suivante, le DIP crée le Réseau d'Éducation Prioritaire (REP) afin d'“encourager la qualité des écoles dans les quartiers populaires" (DIP 2006), en particulier par la mise en place de projets favorisant les apprentissages des élèves en lecture. Avec l'instauration des écoles en REP, des moyens financiers supplémentaires sont attribués et de nouveaux acteurs font leur entrée : un éducateur social avec un rôle de médiateur entre les familles et l'école renforce l'équipe pédagogique (Delay 2013). Pour autant, comme le précisent les autorités scolaires, le principe du partenariat dans le canton de Genève reste modéré, c'est-à-dire fondé sur la bonne volonté des différents acteurs prenant part à ce dispositif institutionnel. Alors qu'en France, la reconnaissance des droits des parents s'inscrit dans la loi d'orientation de 1989 (Périer 2005), à Genève la création de dispositifs de représentation dans l'enseignement primaire (les conseils d'établissement) ne voit le jour qu'en 2008.

Dans le canton de Fribourg, l'intention des autorités scolaires de rapprocher l'école des familles semble encore timide. Le projet de nouvelle loi scolaire (LS) présenté en janvier 2013 reprend l'essentiel des formulations de l'ancienne loi, qui parle de collaboration et d'information. Un alinéa est introduit, surprenant par rapport à la philosophie du partenariat et de la co-éducation : "Les parents se conforment aux attentes de l'école, en particulier aux consignes du corps enseignant. En cas de conflit, ils peuvent s'adresser aux autorités scolaires" (LS 2013). Une autre innovation de la nouvelle loi scolaire fribourgeoise prend par contre la direction du partenariat en prévoyant la création de conseils des parents dans 
les établissements. Cependant, l'alinéa précité laisse présager quelques difficultés dans la concrétisation de ces conseils. À relever aussi, l'obligation récente à Fribourg de l'entrée à l'école à 4 ans, selon l'accord intercantonal sur l'harmonisation de la scolarité obligatoire (Harmos) de 2009 (CIIP 2009). Dans la commune de l'établissement observé, l'introduction de cette année supplémentaire a été effective à la rentrée scolaire 2010-2011.

Si ces deux recherches étudient le rapport des familles à l'école lors des premiers moments de scolarisation, la première à Genève est une étude doctorale dont l'enquête de terrain a débuté en 2011, autour d'une école en REP et de son quartier dit populaire. Elle constitue la toile sur laquelle sont examinées et interrogées les logiques de partenariat ainsi que les interprétations et significations hétérogènes attribuées (explicitement ou non) par les familles et les acteurs scolaires. Les entretiens et observations se sont déroulés dans des lieux et espaces régulés ou codifiés de l'école et du quartier, en particulier dans une des classes à double degré réunissant des élèves de $1^{\text {re }}$ et de $2^{\mathrm{e}}$ année. Une présence régulière de la chercheuse durant huit mois a permis la rencontre de 15 familles majoritairement issues de l'immigration et de milieux défavorisés et de 11 professionnels de l'éducation ( 6 enseignantes, 2 enseignantes chargées de soutien pédagogique, la directrice d'établissement, un éducateur et une enseignante de langue française travaillant avec les mères d'élèves allophones de l'école) appartenant aux nouvelles classes moyennes.

L'étude fribourgeoise a débuté à la rentrée 2012-2013¹, dans un établissement scolaire desservant une population avec une forte proportion de familles immigrantes aux revenus modestes, mais pas uniquement. Dans une visée compréhensive, l'étude cherche à identifier et à rendre compte des processus de construction de la relation entre les familles et l'école dans ses premiers moments, lors de l'entrée à l'école de l'aîné. La relation est approchée dans une perspective de communication interculturelle considérant que les acteurs définissent leur relation à partir de cadres de référence plus ou moins proches négociés dans les interactions. Le corpus de données est constitué selon le principe de triangulation des types de données (observations, entretiens et analyse documentaire). La recherche a lieu dans les quatre classes de $1^{\text {re }}$ et de $2^{\text {e }}$ année de l'école primaire sélectionnée. Elle est centrée sur les familles des enfants, aînés d'une fratrie, qui effectuent leur première année de scolarité. Une des quatre classes étant assurée en duo, l'étude est réalisée auprès de cinq enseignantes.

Les observations ethnographiques directes des premiers entretiens parentsenseignants sont le matériau central de cet article. Ces entretiens sont organisés

I En avril 2014, cette étude a bénéficié d'un subside de deux ans du Fonds National Suisse de la Recherche Scientifique. La première collecte de données a été réalisée avec Angelika Toth (Université de Genève) et Loana Cettou (Université de Fribourg) qui en sont sincèrement remerciées. 
par les enseignantes lors du second trimestre scolaire (mars) à Genève et à la fin du premier semestre (janvier) à Fribourg. 15 entretiens ont ainsi été observés à Genève en 2011 et 21 à Fribourg en 2013. L'intérêt de ce corpus d'observations est de pouvoir saisir les catégories en acte, celles de l'entendement scolaire et familial, qui se manifestent à travers la rencontre formelle, le premier entretien parents-enseignante et guident le travail de collaboration "en train de se faire".

\section{Les entretiens parents-enseignants : performance d'un genre institutionnel}

$\mathrm{D}$ ans leur relation d'une recherche aux États-Unis sur des entretiens formels entre enseignants de premières années d'école et parents migrants mexicains, Howard et Lipinoga (2010) considèrent ces entretiens comme un genre institutionnel, avec lequel les parents sont diversement familiers et pour lequel ils disposent de ressources inégales pour une participation efficace. Les enseignants, semble-t-il, abordent aussi cet exercice imposé avec plus ou moins d'aisance et de ressources, notamment communicationnelles. Howard et Lipinoga se réfèrent à des recherches dans des pays anglo-saxons montrant de fortes similitudes dans la performance de ce genre d'interaction : "une discussion centrée sur les problèmes, une présentation de l'information par l'enseignant dans un registre autoritaire et universalisant, un contrôle asymétrique de l'ordre du jour par l'enseignant et un discours avec une tonalité d'évaluation morale" (35). MacLure \& Walker (2000), analysant des entretiens entre parents et enseignants dans l'enseignement secondaire en Grande-Bretagne, ont comparé le schéma de ces entretiens à celui de consultations entre médecins et patients : communication du diagnostic puis négociation des remèdes à apporter.

Une grande similitude apparaît ici aussi dans le déroulement des entretiens, avec des variations liées à l'interprétation personnelle des enseignantes de cet exercice performatif imposé par l'institution. À Fribourg, l'entretien est invariablement construit autour du bulletin scolaire (document qui suit l'élève tout au long de sa scolarité obligatoire) et de la signature de sa première page, attestant que ce premier entretien formel a bien eu lieu. Si la lourdeur et le formalisme du genre prescrit par l'institution ont été relevés à plusieurs reprises par les enseignantes, le caractère obligatoire de cet échange formel a été aussi souligné par plusieurs d'entre elles dans leur mot de bienvenue aux parents. À Genève, le rituel autour du bulletin scolaire et de sa signature correspond également à la démarche institutionnelle prescrite. La remise du document et l'obligation de le signer rappellent aux parents l'exercice de leurs responsabilités et leur implication dans le 
suivi scolaire de l'enfant. Si le caractère obligatoire de la convocation individuelle n'est pas explicitement souligné par l'enseignante du terrain genevois, il ressort des discours enseignants lorsque le parent ne se présente pas au rendez-vous proposé, précisant qu'il s'agit d'une pratique courante dans cette école.

L'enquête montre ensuite que l'exercice imposé de l'entretien parents-enseignante donne lieu à la communication d'un diagnostic délivré par les enseignantes aux parents. Il s'agit d'une évaluation cachée des apprentissages scolaires et sociaux attendus en première année tels qu'ils sont définis par le Plan d'études romand (CIIP 2002). L'élève est passé au crible par l'enseignante dans "sa capacité ou ses difficultés à apprendre et à se comporter" (Périer 2005, 83). S'écartant des consignes de l'institution qui prescrit pour les élèves de première année un entretien sans évaluation (mais répondant sans doute aux attentes des parents), plusieurs enseignantes du terrain fribourgeois font part de leur diagnostic en se référant aux tableaux d'évaluation des apprentissages des élèves prévus pour la deuxième année. À Genève, si les notes et les orientations sont, à ce stade du cursus scolaire, encore absentes, les évaluations discrètes n'en sont pas moins présentes : l'enseignante a construit ses entretiens autour de quatre axes principaux (relations sociales, apprentissages, jeux, travaux d'évaluations) évoquant les critères de seconde année. Ainsi, ces évaluations communiquées au travers d'un discours diagnostique semblent refléter, dans une certaine mesure, des classements scolaires (Darmon 2001).

Concrètement, la performance de l'entretien formel est aussi un exercice exigeant pour les enseignantes. Son format est généralement de courte durée, souvent calibré dans un espace-temps défini par le calendrier institutionnel (les entretiens ayant lieu à un moment précis de l'année scolaire) et l'organisation individuelle de l'enseignante. Si à Fribourg des variations importantes (entre les enseignantes, mais aussi dans les entretiens réalisés par une même enseignante) s'observent dans leur durée -de 15 à 45 minutes-, à Genève les entretiens formels observés sont calibrés à 30 minutes, temps calculé par l'enseignante en fonction de ses contraintes professionnelles (les entretiens se déroulent à midi et en fin de journée scolaire). Si le principe du partenariat entend la participation de tous les parents et "leur signifier la possibilité de rencontrer les acteurs de l'école [...], il n'est généralement guère tenu compte des contraintes de disponibilité de parents occupant des emplois en horaires décalés" (Périer 2005, 82). La question du choix des moments de rencontre formelle demeure soumise à l'appréciation de chacun, mais en particulier de l'enseignante.

Enfin, l'observation des entretiens met au jour une variation des possibilités d'intervenir données par l'enseignante aux parents. Certains sont très proches du modèle de la consultation médicale décrite par MacLure \& Walker (2010), d'autres donnent plus de place à un dialogue entre l'enseignante et les parents, parfois dès le début de l'entretien, le plus souvent dans la dernière partie uniquement. Les observations montrent toutefois que ce dialogue demeure unidirectionnel et 
ambivalent, en particulier lorsque le diagnostic scolaire fait état de difficultés pour l'élève. Les discours enseignants restent ambigus et laissent peu de place à la réciprocité entre la famille et l'acteur scolaire, notamment lorsque les parents sont éloignés du monde de l'école. Or le partenariat dans son idéal de rapprocher les familles de l'école, notamment dans les interactions avec les enseignants, présuppose que l'ensemble des parents endosse pleinement leurs rôles de partenaires. Ce principe de proximité cherche à valoriser la participation et l'échange, dans un rapport relationnel souhaité égalitaire (Périer 2005). La faible réciprocité entre les familles et l'enseignante, plusieurs fois observée lors de l'échange formel parents-enseignante, laisse penser à l'instar de Périer que ce modèle de relations est plus aisément accessible aux familles pourvues de ressources sociales et culturelles (maîtrisant la langue de scolarisation de leur enfant ainsi que les normes de communication et un code du langage public) que pour celles plus éloignées de la culture légitime "par leur idiome d'origine ou leur façon de parler" $(2005,83)$. L'entretien formel semble moins représenter un dispositif d'échange réciproque parents-enseignante qu'une situation d'interaction paradoxale : convoqué par l'enseignante pour faire le point sur la scolarité de l'enfant, le parent se retrouve dans une situation délicate, voire impossible pour faire entendre sa voix. Si le parent soutient l'enseignante (abonde dans son sens, reconnaît les difficultés de son enfant), c'est lui signifier qu'il joue son rôle de parent collaborant, mais il contribue alors à "enfoncer" (Glasman 2008, 116) son enfant. À l'inverse, s'il le défend (conteste le diagnostic enseignant, réplique vivement), il s'éloigne de son rôle attendu aux côtés de l'institution scolaire. Tout comme d'autres études (Delay 2011), les présentes observations montrent que les familles minoritaires se sont saisies, en partie, du principe des rencontres formelles, mais sans toutefois en maîtriser les enjeux de communication. Peu familières avec le "parler légitime" (Delay 2013, 145), les familles prennent peu la parole, rétorquent peu, restent silencieuses ou hochent de la tête, et attendent généralement la fin de l'entretien ou d'y être invitées pour poser des questions ou faire part d'une anecdote.

\section{Des parents partenaires, avec des devoirs et des conseils à suivre à domicile}

es entretiens parents-enseignants observés consistent d'abord à dresser un état
des débuts de l'enfant dans son parcours d'élève. Un premier diagnostic ins-
titutionnel est communiqué aux parents. Il porte sur les apprentissages scolaires
et sociaux attendus en première année. Lorsqu'arrive l'évocation des préoccu-
pations enseignantes à l'égard de l'élève, celles-ci se projettent souvent sur son 
environnement familial et parfois sur la capacité des familles dans l'éducation de leur enfant. L'entretien devient alors un espace de prescriptions institutionnelles allant jusqu'à vouloir orienter l'action éducative du parent au sein de l'espace domestique. Ce souci de l'école pour l'éducation de l'enfant dans la sphère privée familiale prend la forme de ce qui peut être qualifié de devoirs scolaires et de conseils éducatifs des enseignantes aux parents. Ceux-ci s'observent surtout lorsque la conception du partenariat familles-école repose chez les enseignants (et autres acteurs de l'école) sur une vision déficitaire des familles minoritaires qui provoque une intervention compensatrice (Boulanger et al. 2010), ce qui contredit le principe du partenariat supposant une reconnaissance des contributions réciproques.

Darmon (2001) relève que certains travaux réalisés en classe (dessins, peintures, collages, découpages, graphismes) (r)appellent aux enfants issus de familles proches de la culture scolaire des compétences déjà acquises chez eux, qu'ils peuvent réutiliser ou activer selon les consignes de l'enseignante, ce qui est moins le cas pour des enfants de familles plus éloignées du monde de l'école. Les enseignantes suggèrent ainsi aux parents, aux mères surtout, des activités à effectuer à domicile pour tenter implicitement de rattraper le retard de l'enfant par rapport aux autres : "Faire tous les jours avec [votre enfant] des découpages, du coloriage, etc., pour qu'il s'entraîne et prenne confiance en lui”. Ces devoirs s'apparentent à un processus de "mise en conformité" (Périer 2005, 85) du jeune élève à la norme scolaire comme le suggère cette note d'observation : "L'enseignante oriente la discussion vers le dessin. Elle remarque qu'Alfredo a une tenue de crayon très spéciale et il faudrait qu'il s'entraîne à bien tenir son crayon. Elle se lève pour chercher le cahier de dessins de l'enfant. En le feuilletant devant les parents, elle montre qu'au début Alfredo n'appuyait pas du tout sur le crayon et qu'il y a une évolution importante, mais il y a encore du chemin à faire. Elle demande si les enfants dessinent à la maison. La maman répond que ça n'arrive pas souvent puisque les enfants préfèrent se bagarrer et jouer à d'autres choses. L'enseignante attire l'attention sur les formes peu reconnaissables des dessins de l'enfant. Elle incite les parents à s'entraîner à faire des bonshommes à la maison."

Les recommandations des enseignantes peuvent aussi ressortir d'une tentative de transformation de la morale domestique, de normes éducatives familiales décalées par rapport au modèle éducatif légitime, partagé et défendu spontanément par l'institution scolaire. La question du soutien parental dans les premiers apprentissages scolaires fait l'objet d'attentes ambivalentes et exigeantes. Les enseignantes qui jugent important que le parent encadre et soutienne scolairement son enfant se réfèrent à un "modèle d'encadrement approprié" (Delay \& Frauenfelder 2013, 188) : accompagner l'enfant dans son travail scolaire, mais sans exercer de pressions psychologiques à son égard. Une enseignante, qui perçoit un encouragement insuffisant des parents à l'apprentissage de la lecture par leur fille, redoute aussi l'excès contraire. Elle "montre au père de Lylia le classeur 
d'évaluation avec les exercices réalisés autour des lettres de l'alphabet. Elle lui explique [via un interprète français-albanais] que Lylia n'en connaît que 7 sur les 26 et elle ne connaît pas non plus la lettre Y qui se trouve pourtant dans son prénom. Rire gêné du père. L'enseignante explique que Lylia a fait des progrès mais il y a des 'petites' lacunes [l'enfant rencontre de grandes difficultés en lecture] et c'est peut-être un problème pour l'année prochaine, car les élèves commencent la lecture. Elle demande au père s'il y a des livres à la maison. Celui-ci répond par l'affirmative. L'enseignante insiste sur ce point, la lecture : C'est important, dès tout petit, qu'ils soient dans un univers entouré de livres. Elle ajoute qu'il y $\mathrm{a}$ une bibliothèque à l'école. S'engage ensuite une courte discussion. Le père (s') explique : en 2-3 mois, elle va faire beaucoup de progrès [c'est-à-dire d'ici la fin de l'année], elle va travailler. L'enseignante : Faut pas mettre la pression non plus ! Le père : Non, non. Mais la pression sur la sœur aînée [visiblement en charge du suivi scolaire de Lylia]. C'est bon ? L'entretien prend fin, le père s'en va très vite, le classeur d'évaluation de sa fille sous le bras".

L'ambivalence et l'exigence des normes éducatives portées par l'école, exprimées par les conseils des enseignantes aux parents, concernent aussi l'autonomie et la responsabilisation précoce de l'enfant. Ces valeurs fortement privilégiées dans l'espace scolaire (Bélanger \& Farmer 2012) se retrouvent dans les observations. Critère d'évaluation scolaire par excellence (dès le début de la scolarité), l'autonomie de l'élève est idéalisée par l'école (Dürler 2012). Dans les discours des enseignantes observées se distinguent l'élève autonome, celui qui "s'autogère" lors d'une activité d'apprentissage ou d'un conflit entre enfants (il "sait le gérer tout seul" et s'en extrait) et l'élève qui n'est pas (encore) autonome. Cet enfant est décrit comme étant encore "un peu petit" ou "petit bébé", voire immature par rapport aux comportements prescrits et attendus : gérer son travail (réaliser un exercice seul dans un cadre fixé par l'enseignante), gérer sa tenue (rester "propre", se rendre aux toilettes seul). Là aussi, l'autonomie est valorisée par les acteurs de l'école pour autant qu'elle se décline sans excès. L'autonomie maximale n'est pas souhaitée : le respect des règles de vie de la classe et de l'école est un autre critère important d'appréciation des élèves, qui contrebalance l'autonomie. Le discours enseignant peut donc paraitre contradictoire aux parents, invités à soutenir le développement de l'autonomie de leur enfant, mais aussi son aptitude à se conformer aux règles de vie.

Dans leurs discours aux parents, les enseignantes relativisent souvent la pertinence de cette attente d'autonomie enfantine en première année. À Fribourg, elles font allusion à l'introduction récente de l'obligation scolaire à 4 ans et suggèrent parfois que des enfants trop jeunes sont contraints d'aller à l'école, comme elles-mêmes le sont de scolariser des enfants qui ne sont pas encore prêts. Elles accueillent avec reconnaissance un atelier dit d'éducation familiale, offert par les autorités scolaires, dont l'objectif est de soutenir les parents dans la préparation de l'enfant à l'entrée à l'école. 
Cependant, si toute mesure de soutien des parents est à saluer, n'y a-t-il pas là un risque d'accréditer l'idée que les familles devraient livrer à l'école leur enfant déjà équipé et fonctionnel une année plus tôt qu'avant ? Quelle est alors l'utilité pour l'égalisation des chances de réussite de l'avancement de la scolarisation à 4 ans ? Les observations indiquent que la responsabilité de la mise en conformité de l'élève à la norme scolaire échoit avant tout aux familles (qui ont à Fribourg une année de moins pour y parvenir), plutôt qu'à l'école (qui bénéficie quant à elle d'une année supplémentaire).

\section{Valses-hésitations autour des prescriptions institutionnelles sur le bien-être de l'enfant}

$\mathrm{H}$

étérogènes, les préconisations enseignantes touchent à de nombreux domaines de la sphère intime des familles, accordant la prépondérance au bien-être de l'enfant. C'est l'“habitus global” de l'enfant (Frauenfelder \& Delay 2005,84 ) -saisi dans sa dimension psychologique, sociale et sanitaire- qui fait ici l'objet du diagnostic institutionnel, le mandat des acteurs scolaires ne se limitant plus aux apprentissages scolaires. La prise en considération du bien-être de l'enfant pour un "mieux-être" (Giuliani 2009, 85) puis la délivrance de conseils s'observent à nouveau lorsque prédomine chez les enseignantes une vision déficitaire des familles minoritaires (Boulanger et al. 2010). Les enseignantes formulent ainsi des conseils relatifs à l'état de santé de l'enfant, à son alimentation. Le goûter, ce fragment de la maison apporté et consommé à l'école, est un exemple souvent évoqué par les enseignantes observées. L'enseignante évoque ainsi un souci avec les goûters des récréations. Elle ne le dit pas explicitement à la mère, mais Mariya a souvent des chips pour le matin à 10 h. ce qu'elle juge inadapté. De plus, Mariya oublie de manger son en-cas pendant les récréations ou elle l'oublie à la maison. Si Mariya est "tête en l'air", l'enseignante suggère à la mère de demander à sa fille après l'école, si elle a mangé son goûter ou non. La mère gênée avoue à demi-mot en quoi consiste le repas du soir de sa fille : "C'est un secret, mais Mariya boit un biberon avec du chocolat!”.

Le matériau qualitatif permet de relever que l'équilibre d'un goûter concerne aussi les boissons, comme on peut le voir dans cette autre observation : "L'enseignante enchaîne avec la routine de la classe et explique aux parents que dans la classe, ils font un goûter commun tous les après-midis. Les enfants apportent des fruits et des légumes et ils boivent de l'eau. Elle insiste sur la boisson, précisant bien que les parents ne doivent pas acheter des boissons sucrées, puisqu'il y a de l'eau dans la classe". Cette préconisation, en apparence anodine, souligne le souci enseignant d'une alimentation saine de l'enfant et plus globalement de 
sa santé. Au-delà de leur préoccupation immédiate (une bonne nutrition favorise la concentration et la performance dans le travail scolaire), les enseignantes s'acquittent d'une mission prescrite par l'école, mandatée par la société (DICS 2008). Le bien-être de l'enfant, défini dans la perspective de l'école reflétant celle de la société majoritaire, est implicitement présenté comme une norme partagée par tous ou devant l'être à terme si les parents appliquent les pratiques qui leur sont si fortement recommandées. Aucune place n'est donnée à la négociation, à l'échange sur les conceptions des uns et des autres quant à ce qui est bon pour l'enfant. Il y a d'un côté ceux qui savent et de l'autre ceux qui sont encore ignorants.

S'inquiétant du bien-être global de l'enfant, les enseignantes suggèrent des conseils éducatifs aux parents à suivre au sein de la sphère privée familiale. Destinés à aider les parents dans leur rôle de partenaires de l'école, parfois à anticiper des difficultés pressenties par les enseignantes, ils sont surtout formulés lorsque les enseignantes perçoivent une contradiction entre les pratiques éducatives des parents et les leurs, ce qui rend difficile à leurs yeux la construction d'une "complémentarité idéale des rôles" (van Zanten 2001, 155) entre parents et enseignants. L'entretien formel entre enseignante et parents devient un espace de prescriptions institutionnelles pouvant aller jusqu'à vouloir orienter l'action éducative du parent dans la sphère privée, l'école cherchant à influencer à distance l'organisation et le contenu de la vie des familles (Terrail 1997). Si les familles proches socialement et culturellement du monde de l'école disposent de ressources (notamment communicationnelles) pour affronter le regard parfois intrusif des enseignantes dans leur vie privée, les plus éloignées de la culture scolaire, moins armées face à cette immixtion, peuvent vivre des tensions et des incompréhensions vives et se sentir disqualifiées dans leurs conceptions éducatives (Delay \& Frauenfelder 2013). Les échanges formels deviennent un espace de tensions liées à la division du travail éducatif (Payet et al. 2011) où se joue, se négocie et se définit le bien-être de l'enfant. Les enseignantes se trouvent alors dans une position délicate, car émettre une recommandation, c'est mettre en cause les compétences éducatives parentales, voire un mode de vie perçu comme peu adéquat. Ainsi, les enseignantes sont parfois réticentes à formuler des conseils. Prises dans des tensions normatives, elles sont tiraillées entre ce qu'elles considèrent être le bien de l'enfant, corroborant un souci institutionnel croissant pour la sphère privée familiale, et le respect des familles, de leur autonomie et de leur responsabilité en tant que parents.

\section{Conclusion}

L'entretien parents-enseignante est un genre institutionnel particulier : prescrit par l'école aux professionnels de l'éducation comme aux familles, le script n'en demeure pas moins diversement connu et partagé par les acteurs. S'agit-il simplement d'un compte rendu que l'enseignante fait aux parents des performances 
de leur enfant dans la classe ? Ou est-ce aussi le lieu de mise en place d'un partenariat entre ces deux instances de socialisation que sont la famille et l'école ? Les observations des premiers entretiens parents-enseignants dans des classes de $1^{\text {re }}$ et de $2^{\text {e }}$ année d'école de deux établissements, Genève et à Fribourg, montrent que l'échange est ambivalent et reste unidirectionnel. Si le principe du partenariat se comprend comme une nouvelle norme institutionnelle de "faire ensemble" -école et familles- au travers d'un travail de collaboration et d'entente mutuelle, autrement dit dans un rapport relationnel égalitaire (Périer 2005), ce premier entretien supposerait un dialogue bidirectionnel ou, du moins, une réciprocité entre les familles et l'enseignante. Or l'échange est d'abord l'occasion pour l'enseignante de parler aux parents de leur enfant dans sa classe. L'enfant dans sa famille n'a pas de véritable place dans l'entretien, ce qui permettrait pourtant d'enrichir la compréhension qu'en a l'enseignante et de valoriser le rôle éducatif du parent partenaire. Tout comme le relèvent Payet et al. (2011), cet entretien formel n'est pas non plus un lieu où l'enseignante présenterait aux parents son projet pédagogique. Ce premier entretien constitue ainsi un espace dialogique inégal, en tout cas pour les familles peu familières de la culture scolaire.

L'analyse montre que les enseignantes vont généralement au-delà du simple compte rendu : elles orientent les entretiens vers l'avenir en suggérant aux parents des tâches scolaires à effectuer à domicile avec leurs enfants et se risquent à quelques conseils éducatifs. De ce point de vue, l'entretien représente un espace de prescriptions institutionnelles en particulier si les préconisations ont "pour finalité première de compenser les carences éducatives attribuées aux parents [de milieux défavorisés]" (Boulanger et al. 2010, 152). Cependant les enseignantes formulent souvent des recommandations timidement, voire avec réticence, prises dans une tension normative entre le souci du bien de l'enfant et celui de ne pas empiéter sur le domaine privé des familles. Jusqu'où peuvent-elles aller ? Jusqu'où les parents peuvent-ils ou sont-ils prêts à les laisser avancer? Le premier entretien met en évidence une troisième dimension de cet échange ambivalent, un espace de tensions liées à la division du travail éducatif.

Enfin, ces recommandations enseignantes se transforment parfois en injonctions complexes, implicites ou contradictoires pour les parents, participant à une forme d'interpénétration des espaces de socialisation entre la sphère privée (les familles) et celle publique (l'école). Ces entretiens sont ainsi le lieu par excellence où se négocie la bonne distance entre la famille et l'école : du point de vue de la délimitation des espaces de compétences et de la négociation des identités (individuelles, familiales). Un travail d'explicitation et de métacommunication sur les attentes et objectifs des uns et des autres vis-à-vis de ces entretiens, tout comme sur les rôles respectifs dans l'éducation de l'enfant, permettrait aux parents comme aux enseignantes de trouver la bonne distance et de faire de ces entretiens un apport précieux à la construction d'une base solide d'un partenariat entre eux. 


\section{Références bibliographiques}

BLOOM B.S. 1981 All our children learning, New York, McGraw Hill Book Co

BÉLANGER N. \& FARMER D. 2012 "Autonomie de l'élève et construction de situations scolaires. Études de cas à l'école de langue française en Ontario (Canada)”, Éducation et Sociétés-29, 173-191

BOULANGER D., LAROSE F. \& COUTURIER Y. 2010 "La logique déficitaire en intervention sociale auprès des parents : les pratiques professionnelles et les représentations sociales", Nouvelles pratiques sociales-23, 152-176

CHAMBOREDON J.-C. \& PREVOT J. 1973 "Le 'métier d'enfant'. Définition sociale de la prime enfance et fonctions différentielles de l'école maternelle”, Revue française de sociologie-14(3), 295-335

CIIP (Conférence intercantonale de l'instruction publique de la Suisse romande et du Tessin) 2009, <www.edk.ch/dyn/11737.php>, consulté le 30-06-2014

CIIP (Conférence intercantonale de l'instruction publique de la Suisse romande et du Tessin) 2003 Déclaration de la CIIP relative aux finalités et objectifs de l'École publique, Neuchâtel

CIIP (Conférence intercantonale de l'instruction publique de la Suisse romande et du Tessin) 2002, <www.ciip.ch/CMS/default.asp?ID=1298>, consulté le 30-06-2014

DARMON M. 2001 "La socialisation, entre famille et école. Observation d'une classe de première année de maternelle", Sociétés et Représentations-11, 517-538

DICS (Direction de l'instruction publique, de la culture et du sport) $2008<$ www.fr.ch/ dsas/files/pdf36/402_110922_Ct_FR_concept_sant-cole_def_F1.pdf>, consulté le 3006-2014

DIP (Département d'instruction publique) 2006 <www.ge.ch/enseignement_primaire/rep/ welcome.asp>, consulté le 30-06-2014

DIP (Département d'instruction publique) 2005 Les treize priorités pour l'instruction publique genevoise, République et canton de Genève

DELAY C. 2013 "L'impératif scolaire du partenariat et son appropriation partielle au sein des familles populaires : un exemple genevois”, Éducation et Sociétés-32, 139-154

DELAY C. 2011 Les classes populaires à l'école, Rennes, PUR

DELAY C. \& FRAUENFELDER A. 2013 “Ce que 'bien éduquer' veut dire. Tensions et malentendus de classe entre familles et professionnels de l'encadrement (école, protection de l'enfance)", Déviance et Société-37, 181-206

DUBET F. 1997 École, familles : le malentendu, Paris, Éditions Textuel

DÜRLER H. 2012 "Les dispositifs scolaires de fabrication de l'autonomie : implications et contradictions", Colloque Sociologie et didactiques : vers une transgression des frontières, Lausanne, Haute École Pédagogique

ELBERS E. \& DE HANN M. 2014 "Parent-teacher conferences in Dutch culturally diverse schools: Participation and conflict in institutional context", Learning, Culture and Social Interaction, sous presse

FIELD S., KUCZERA M. \& PONT B. 2007 En finir avec l'échec scolaire : Dix mesures pour une éducation équitable, Paris, OCDE 
FRAUENFELDER A. \& DELAY C. 2005 "La cause de l'enfant et sa résonnance spécifique auprès des classes moyennes à travers la régulation du problème de 'maltraitance"', Carnet de bord-10, 79-92

GLASMAN D. 2008 L'institution scolaire et les parents de milieux populaires : habilitation ou disqualification ?, in Payet J.-P., Giuliani F. \& LaForgue D. dir. La voix des acteurs faibles. De l'indignité à la reconnaissance, Rennes, PUR, 107-119

GLASMAN D. 1992 L'école réinventée? Le partenariat dans les zones d'éducation prioritaires, Paris, L'Harmattan

GIULIANI F. 2009 "Éduquer les parents ? Les pratiques de soutien à la parentalité auprès des familles socialement disqualifiées", Revue française de pédagogie-168, 83-92

HENDERSON A.T. \& MAPP K.L. 2002 A new wave of évidence: The impact of school, family, and community connections on student achievement. Annual synthesis, 2002, Austin, TX, Southwest Educational Development Laboratory

HOWARD K.M. \& LIPINOGA S. 2010 "Closing down openings: Pretextuality and misunderstanding in parent-teacher conferences with Mexican immigrant families", Language E Communication-30(1), 33-47

HOOVER-DEMPSEY K.V., BATTIATO A.C., WALKER J.M.T., REED R.P., DEJONG J.M. \& JONES K.P. 2001 "Parental Involvement in Homework", Educational Psychologist-36(3), 195-209

HUMBEECK B., LAHAYE W., BALSAMO A. \& POURTOIS J.-P. 2006 "Les relations école-famille : de la confrontation à la coéducation", Revue des sciences de l'éducation-32(3), 649-664

JAEGGI J.-M., OSIEK F. \& FAVRE B. 2003 Familles, école et quartier, de la solitude au sens. Échec ou réussite scolaire d'enfants en milieu populaire, Genève, SRED

KHERROUBI M. dir. 2008 Des parents dans l'école, Ramonville-Saint-Agne, Erès

LÉGER A. \& TRIPIER M. 1986 Fuir ou construire l'école populaire ? Paris, Méridiens Klincksiek

LS (Présentation du projet de nouvelle Loi scolaire) 2013 <www.fr.ch/ww/fr/pub/actualites.cfm?fuseaction_pre=Detail\&NewsID=43227>, consulté le 30-06-2014

MANGEZ E., JOSEPH M. \& DELVAUX B. 2002 Les familles défavorisées à l'épreuve de l'école maternelle. Collaboration, lutte, repli, distanciation, Université catholique de Louvain, CERISIS

MACLURE M. \& WALKER B.M. 2000 "Disenchanted Evenings: The Social Organization of Talk in Parent-Teacher Consultations in UK Secondary Schools", British Journal of Sociology of Education-21(1), 5-25

ODM (Office fédéral des migrations) \& CFM (Commission fédérale pour les questions de migration) 2012 Encouragement de l'intégration dans le domaine préscolaire. Enfants de 0 à 4 ans, parents, professionnels et personnes de référence. Encouragement de l'intégration des étrangers. Programme des points forts 2008-2011. Projet pilote. Projet d'évaluation version succincte, Lucerne, Institut pour l'école et l'hétérogénéité, Haute École Pédagogique de Suisse centrale

PAGNOSSIN E. 2010 La recherche suisse romande en éducation et en formation (2007-2009), Neuchâtel, IRDP 
PAYET J.-P., SANCHEZ-MAZAS M., GIULIANI F.E. \& FERNANDEZ R. 2011 "L'agir scolaire et régulations et incertitudes. Vers une typologie des postures enseignantes de la relation à autrui", Éducation et Sociétés-27, 23-37

PAYET J.-P. 1995 Collèges de banlieue. Ethnographie d'un monde scolaire, Paris, Méridiens Klincksieck

PÉRIER P. 2007 Des élèves en difficulté aux parents en difficulté : le partenariat école/familles en question, in Toupiol G. éd. Tisser des liens pour apprendre, Paris, Retz, 90-107

PÉRIER P. 2005 École et familles populaires. Sociologie d'un différend, Rennes, Presses universitaires de Rennes

PERREGAUX C., CHANGKAKOTI N., HUTTER V. \& GREMION M. 2008 "L'accueil scolaire d'élèves nouvellement arrivés en Suisse : tensions entre séparation et inclusion", Glottopol, revue de sociolinguistique en ligne, 96-109

PERROTON J. 2000 "Les ambiguïtés de l'ethnicisation des relations scolaires : l'exemple des relations école-famille à travers la mise en place d'un dispositif de médiation”, VEI Enjeux-121, 130-147

PITHON G., ASDIH C. \& LARIVÉE S.J. 2008 Construire une "communauté éducative". Un partenariat famille-école-association, Bruxelles, De Boeck Université

POWELL D.R, SON S.-H., FILE N. \& SAN JUAN R.R 2010 "Parent-school relationships and children's academic and social outcomes in public school pre-kindergarten", Journal of School Psychology-48(4), 269-292

QUEIROZ (de) J.-M. 2000 "Les remaniements de la 'séparation scolaire”, Revue Française de Pédagogie-133, 37-48

RAYNA S. 2010 Parents-professionnels : la coéducation en questions, Ramonville-SaintAgne, Erès

RIMM-KAUFMAN S.E. \& PIANTA R.C. 2000 "An ecological perspective on the transition to kindergarten. A theorical framework to guide empirical research", Journal of Applied developmental psychology-21(5), 491-511

STAMM M., REINWAND V., BURGER K., SCHMID K., VIELHAUSER M. \& MUHEIM V. 2009 Éducation de la petite enfance en Suisse. Étude de base élaborée à la demande de la Commission suisse pour l'UNESCO, Berne, CNSU

TERRAIL P. 1997 Sociologie des interactions Famille/École, Sociétés Contemporaines-25, 67-83

THIN D. 1998 Quartiers populaires. L'école et les familles, Lyon, Presses universitaires de Lille

van ZANTEN A. 2001 L'école de la périphérie. Scolarité et ségrégation en banlieue, Paris, Presses universitaires de France

VATZ-LAAROUSSI M., KANOUTÉ F. \& RACHÉDI L. 2008 "Les divers modèles de collaborations familles immigrantes-écoles : de l'implication assignée au partenariat", Revue des sciences de l'éducation-34(2), 291-311 